\title{
Assessment of passive respiratory mechanics in infants: double versus single occlusion?
}

\author{
I. Goetz, A.F. Hoo, S. Lum, J. Stocks
}

\begin{abstract}
Assessment of passive respiratory mechanics in infants: double versus single occlusion? I. Goetz, A.F. Hoo, S. Lum, J. Stocks. (C) ERS Journals Ltd 2001.

ABSTRACT: The single breath or occlusion technique (SOT) is widely used to assess passive respiratory mechanics in infants, but depends on various underlying assumptions. Recently, it has been proposed that such measurements could be internally validated by performing two brief airway occlusions during the same expiration. The aim of this study was to evaluate the use of the double occlusion technique (DOT) using a new commercially available program (Jaeger MasterScreen BabyBody Erich Jaeger GmbH, Würzburg, Germany).

Paired measurements of respiratory system compliance $\left(C_{\mathrm{rs}}\right)$ and resistance $(R \mathrm{rs})$ using both SOT and DOT were obtained in 18 healthy sedated infants (age range 4-41 weeks, weight $2.7-9.9 \mathrm{~kg}$ ).

There was close agreement between both methods of assessing $C_{r s}$ in all infants, the mean within-subject difference $(95 \%$ confidence interval $(C I))$ for DOT-SOT being $\mathbf{- 0 . 0 6}(-0.55-+0.42) \mathrm{mL} \cdot \mathrm{kPa}^{-1} \cdot \mathrm{kg}^{-1}$. By contrast, estimates of $R \mathrm{rs}, \mathrm{DO}$ were on average $20 \%$ lower than those for $R \mathrm{rs}, \mathrm{SO}$, (mean within-subject difference $(95 \% \mathrm{CI})$ being $\mathbf{- 0 . 6 7}$ $\left.(-1.04--0.31) \mathrm{kPa}^{-1} \cdot \mathrm{L}^{-1} \cdot \mathrm{s} ; \mathrm{p}<0.01\right)$.

The relatively lower values obtained for $R$ rs,DO may reflect the higher mean lung volume at which it was calculated. Further work is required to investigate the clinical and epidemiological relevance of this new approach, and whether there are any advantages of using both techniques when assessing passive mechanics in infants.

Eur Respir J 2001; 17: 449-455.
\end{abstract}

Portex Anaesthesia, Intensive Therapy and Respiratory Unit, Institute of Child Health, London, UK.

Correspondence: I. Goetz, Portex Anaesthesia, Intensive Therapy an Respiratory Unit, Institute of Child Health, 30 Guilford Street, London, UK.

Fax: 442078298634

Keywords: Infan

respiratory function tests respiratory mechanics

Received: September 82000

Accepted after revision April 232000
The single and multiple occlusion techniques are simple, noninvasive means of assessing the mechanical properties of the respiratory system in human infants. When using the single breath or occlusion technique (SOT), respiratory compliance $(C \mathrm{rs})$, resistance $(R \mathrm{rs})$ and the expiratory time constant ( $t \mathrm{rs})$ can be assessed [1 -3], whereas the multiple occlusion technique (MOT) [4] only allows the measurement of $C$ rs. The validity of both techniques is highly dependent on two underlying assumptions, namely complete absence of respiratory muscle activity and rapid pressure equilibration throughout the entire respiratory system, during the airway occlusion. In addition, the MOT requires a stable end-expiratory level (EEL) and may be relatively time consuming to perform, since numerous different occlusions have to be performed at different expired volumes. While potentially quicker to perform, the SOT is dependent on complete relaxation throughout the entire expiration following release of the occlusion, and the ability to represent the respiratory system by a single time constant, a condition not easily realised in the presence of severe lung or airway disease.

It has been suggested that results can be internally validated by performing both the MOT and SOT within the same child, with confidence in the results being increased if values of $C_{\mathrm{rs}}$ from the two techniques agree closely $[3,5]$. While this has proved to be a valuable approach, as mentioned previously, the accuracy of the MOT is highly dependent on a stable EEL, and the fact that it is rather time consuming, may limit its application. Alternative approaches that have been proposed to increase the reliability of measurements include the multiple interruption technique (MIT), which potentially overcomes the problems of an unstable EEL, by obtaining multiple pairs of volume-pressure data during a single breath [6], and the double occlusion technique (DOT) which is proposed to perform two occlusions within the same or the subsequent breath, in order to relate changes in volume to those of pressure, independently of the volume baseline [7]. However, both of these techniques were only designed to measure $C_{\mathrm{rs}}$ and give no information about $R$ rs or $t$ rs, and successful measurements using the MIT are difficult to achieve in infants with a rapid respiratory frequency.

An alternative approach could be to extend the analysis originally described for the DOT, by relating the flow immediately prior to the second occlusion to the elastic recoil pressure, measured at the airway opening during relaxation, against this occlusion, in order to measure Rrs. Thus, by performing two occlusions at suitably spaced intervals during expiration, one could potentially obtain a measure of both $C$ rs and $R \mathrm{rs}$, which were independent both of the EEL and any assumptions regarding the respiratory system being represented by a single compartment model. 
The aim of this study was to evaluate this new adaptation of the DOT in comparison with the SOT, using a new commercially available program.

\section{Materials and methods}

\section{Equipment and software}

Measurements were performed using the Resistance/ Compliance (RC) program of the Jaeger MasterScreen BabyBody (V. 4.5 Erich Jaeger GmbH, Würzburg, Germany) collecting data at sampling frequency of $500 \mathrm{~Hz}$. The experimental set up consisted of a pneumotachometer (PNT; Paed-PT S Erich Jaeger $\mathrm{GmbH}$ ) with a solid state pressure sensor measuring a flow range of $\pm 1500 \mathrm{~mL} \cdot \mathrm{s}^{-1}$ (resolution $1 \mathrm{~mL} \cdot \mathrm{s}^{-1}$, accuracy $\pm 3 \%$ ) and a volume range of $\pm 3000 \mathrm{~mL}$ (resolution $0.1 \mathrm{~mL}$ ). The linearity of the PNT was checked and found to be linear within $\pm 3 \%$ over a flow range of $0-40 \mathrm{~L} \cdot \mathrm{min}^{-1}$. The PNT was slotted into a pressure sensor housing in which two solid state pressure sensors, the flow pressure sensor (SCP004D, range $\pm 1 \mathrm{kPa}$ ) and the airway opening pressure sensor (SX01 $\overline{\mathrm{D}}$, range $\pm 6.9 \mathrm{kPa}$ ), were mounted to allow simultaneous measurement of flow $\left(V^{\prime}\right)$ and pressure at the airway opening $(P \mathrm{ao})$. A low deadspace shutter with an inflatable latex balloon (volume $0.7 \mathrm{~mL}$ ), which was operated by compressed air generated by a pump, was connected to the PNT. The other outlet of the PNT was connected directly to a transparent facemask. The total deadspace and resistance of the PNT and shutter were $6.8 \mathrm{~mL}$ and $0.38 \mathrm{kPa} \cdot \mathrm{L}^{-1} \cdot \mathrm{s}$, respectively. Use of a size 2 Jaeger mask added an extra $7.5 \mathrm{~mL}$ deadspace (calculated as $50 \%$ of its water displacement $[8,9]$ ).

Linearity checks of flow (and therefore volume which is integrated from flow) were undertaken with a KDG 1100 Flowmeter (KDG Flowmeters, Sussex, UK), which demonstrated that the PNT plus shutter had a linear response $( \pm 2 \%)$. A P200U Digitron Manometer (Digitron Instrumentation Ltd, Hartford, UK) was used to check the PNT recordings which were shown to be accurate over the range of $0-4 \mathrm{kPa}( \pm 2 \%)$.

During validation of the program, all raw data were recorded as American Standard Code for Information Interchange (ASCII) files during data collection. All algorithms used for automatic calculation of the parameters were then checked manually by recalculating these parameters from the appropriate changes in pressure, flow and volume measured by cursor placement on the ASCII traces.

In addition, these ASCII files were imported into a well established and fully validated lung function data analysis program (Squeeze [10], [11]). There was no significant difference (maximal $2 \%$ ) between the online calculation with the Jaeger programme of tidal volume $(V \mathrm{~T})$, flow $\left(V^{\prime}\right)$ and respiratory frequency $(f \mathrm{R})$ compared with the "Squeeze" analysis of the corresponding ASCII-files.

\section{Subjects}

Measurements were made in infants recruited to an epidemiological study [12], who were being studied at $\sim 6$ weeks and 6 months of age. Informed written parental consent was obtained for all studies. The study was approved by the East London and City Research Ethics Committee.

Measurements of passive respiratory mechanics using both the SOT and DOT were attempted in 34 fullterm healthy infants. All infants were healthy on the day of test and none had had respiratory symptoms for at least three weeks before the measurements.

\section{Protocol}

The infants were physically examined prior to sedation with Chloral hydrate syrup $\left(50-100 \mathrm{mg} \cdot \mathrm{kg}^{-1}\right.$ bodyweight), which was followed by a feed. Oxygen saturation $\left(S_{\mathrm{a}}, \mathrm{O}_{2}\right)$ and cardiac frequency $\left(f_{\mathrm{c}}\right)$ were recorded throughout the procedure. As soon as the infant was asleep, a small transparent mask was fitted around the nose and mouth, using therapeutic silicone putty to achieve an airtight seal. All measurements were performed in the supine position with the infant's neck being slightly extended. Infants who remained in quiet sleep after completion of the lung function protocol for the epidemiological study were included in this methodological comparison of the DOT versus the SOT. In such infants, the mask was attached to the Jaeger PNT, which was then zeroed to ensure a stable volume signal. Measurements of passive respiratory mechanics, using both the SOT and DOT were then performed in a random order as described later.

Once recordings commenced, the pattern of tidal breathing was observed until it was noted to be regular and the child appeared to be in quiet sleep [13]. At least 6 breaths of regular tidal breathing were recorded before activating an occlusion to occur during the following breath, either at end-inspiration (SOT) or at both end-inspiration and mid-expiration (DOT). Midexpiratory volume was calculated as $50 \%$ of the mean expired volume from 5 breaths prior to each occlusion. After the occlusion, at least 6 further tidal breaths were recorded to ensure that the end-expiratory level (EEL) had been re-established without evidence of leak $[3,14]$. After each successful occlusion, results were automatically calculated and displayed.

The option for off-line analysis allowed subsequent modification of these automatically calculated parameters when required for quality control. The algorithms for calculating $C_{\mathrm{rs}}$ and $R \mathrm{rs}$ were as follows:

Single occlusion. Crs: Volume (V)/Pressure $(P)$;

$$
C_{\mathrm{rs}, \mathrm{SO}}=\frac{V \text { ext }}{P_{1}}
$$

where $V$ ext is the extrapolated expiratory portion of the flow/volume loop to zero flow (fig. 1) and $P 1$ is the mean pressure at the airway opening during relaxation (as shown by a plateau) against an end-inspiratory occlusion; i.e. elastic recoil pressure of the respiratory system (fig. 1); Rrs: time constant/Crs;

$$
R \mathrm{rs}, \mathrm{SO}=\frac{t_{\mathrm{rs}}}{C_{\mathrm{rs}}}-R \mathrm{app}
$$

where $t \mathrm{rs}$ is $V / V^{\prime}$ as calculated from the regression of the 


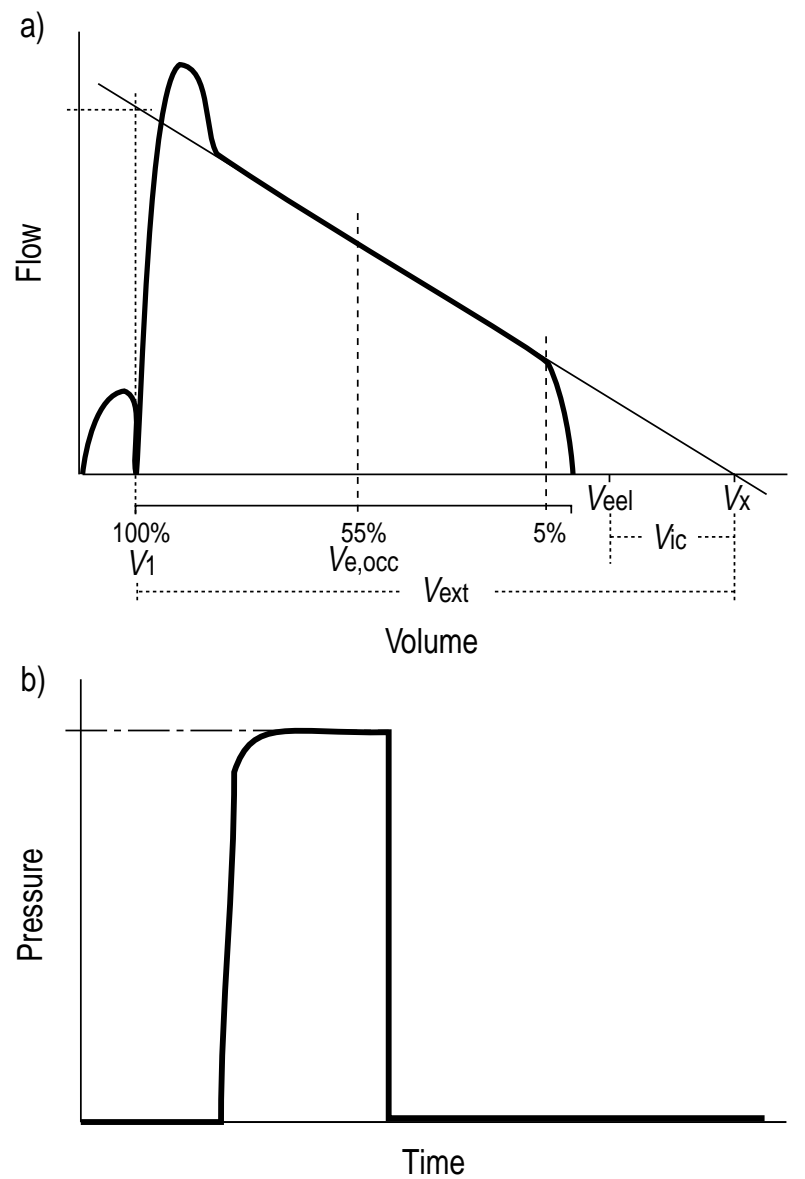

Fig. 1. - Single occlusion technique: a) Flow/volume trace and b) pressure/time trace ----: intersections with flow/volume curve indicating $\mathrm{Vol} \% \mathrm{~A}$ and $\mathrm{Vol} \% \mathrm{~B}$ (limits of linear regression analysis for calculation of flow/volume regression line $(t \mathrm{rs})$; default setting set to analyse over $55-5 \%$ volume remaining in the lung); $\cdots$. axes intersections indicating pseudoflow ( $V^{\prime}$ ext $)$ at moment of airway occlusion, obtained by back extrapolation of $t_{\mathrm{rs}}$; $V_{\mathrm{x}}$ : forward extrapolation of $t$ rs to zero flow, representing the volume to which the infant would have expired passively if a premature inspiratory effort had not occurred (i.e. elastic equilibrium volume); $V$ eel: mean end-expiratory level of the 5 breaths preceding the occlusion; $V_{\text {ic: }}$ volume intercept, representing the extent to which functional residual capacity is dynamically elevated; $V_{1}$ : volume in the lung at moment of occlusion; $V_{\text {ext: }}$ extrapolated expired volume, i.e. the volume of air in the lung at moment of occlusion above the passively determined elastic equilibrium volume (i.e. $\left.V_{1}-V_{\mathrm{x}}\right) ; V_{\mathrm{e}}$,occ: expired volume after release of the occlusion; - - _ : intersection with pressure axis indicating elastic recoil pressure at time of occlusion $(P 1)$.

descending expiratory portion of the flow/volume loop and Rapp is the resistance of the apparatus.

\section{Double occlusion.}

$$
C_{\text {rs, DO }}=\frac{V_{1}-V_{2}}{P_{1}-P_{2}}
$$

where $V_{1}$ and $V_{2}$ represent the volume above EEL remaining in the lung during the first and second occlusion, and $P_{1}$ and $P_{2}$ are the pressures at the airway-opening during each of these occlusions.

$$
R_{\mathrm{rs}, \mathrm{DO}}=\frac{P_{2}}{V^{\prime} 2}-R \mathrm{app}
$$
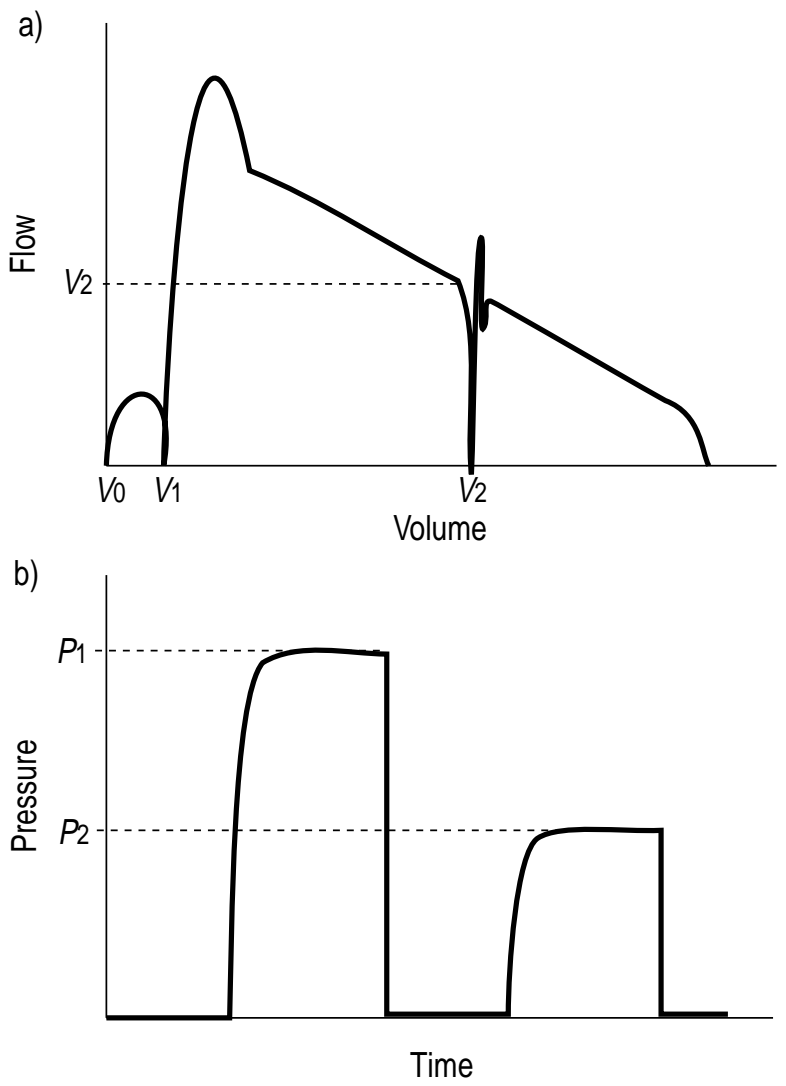

Fig. 2. - Double occlusion technique: a) Flow/volume trace and b) pressure/time trace. $V_{1}, V_{2}$ and $P_{1}, P_{2}$ represent the volume above end-expiratory level $(V)$ and elastic recoil pressure $(P)$ at time of first and second occlusion, respectively. $V^{\prime} 2$ is the flow immediately prior to the 2 nd occlusion.

where $V^{\prime} 2$ is the expiratory flow measured immediately before the second occlusion (fig. 2).

\section{Criteria for technically acceptable occlusions}

For the SOT the default setting was set to accept manoeuvres as being satisfactory if: 1) flow/volume curves had an expiratory portion in which a linear regression line through $50 \%$ of expired volume (preset to analyse over the range $55 \%-5 \%$ volume remaining in the lung above the EEL; fig. 1) could be fitted with a coefficient of determination $\left(\mathrm{r}^{2}\right)$ of at least 0.99: 2) pressure at the airway opening attained a plateau (fig. 1); pressure changes during the period of occlusion were automatically calculated and a plateau was deemed to have occurred if $P$ ao was maintained for at least $100 \mathrm{~ms}$ with a SD of $\leqslant 10 \mathrm{~Pa}$ and a deviation between start and end of this period of $<2 \%$; provided $\geqslant 400 \mathrm{~ms}$ had elapsed (which, by experience, was found to be the minimum duration of occlusion to ensure true pressure equilibration in the majority of babies), the occlusion was released automatically once the program recognised a satisfactory pressure plateau, or after a maximum occlusion time of $1200 \mathrm{~ms}$.

For the double occlusion technique, automatic recognition of technically satisfactory manoeuvres 
demanded the same criteria for both pressure plateaux, except that, during the second occlusion, the duration of the plateau needed to be $\geqslant 70 \mathrm{~ms}$.

During data collection, trials were not discarded even if automatic error messages regarding poor quality of the pressure plateaux or flow/volume curves were displayed, since subsequent (off-line) manual adaptations (e.g. to the precise portion of the curve over which regression had been fitted), sometimes enabled a trial that would have been automatically discarded by the program to be saved. On the other hand, even if all the automated criteria had been fulfilled, trials accepted by the program sometimes had to be rejected after visual inspection, if for example active expiration, slow equilibration or early inspiration had occurred.

The main causes of failure to obtain acceptable data were: 1) alinear flow/volume relationship during passive expiration (SOT only); 2) evidence of expiratory braking or active expiration; 3) failure to attain a relaxed $P$ ao plateau; 4) early inspiration after first or second (DOT only) occlusion; 5) failure to sleep or arousal due to the manoeuvre. Attempts were made to obtain $\geqslant 3$ (minimum 2 if $C$ rs and $R$ rs values were within $10 \%$ of each other) technically valid occlusions for both techniques in each infant.

\section{Statistical analysis}

Statistical analysis of the data were performed using the statistical package for the social sciences (SPSS) for windows (v 8.0). Within subject comparison of $C$ rs and $R$ rs derived from the SOT and DOT was performed using the method of Bland and Altman [15, 16] whereby the mean difference between the two methods was plotted against the mean of the two measurements to visually clarify the outcome.

To assess whether results would be biased in any way by the number of valid manoeuvres available for each infant, within subject comparisons of SOT and DOT were performed using the results from each infant that were derived from the mean of 1) all technically acceptable trials, 2) the first two such trials from both methods and 3) the first five acceptable trials from each technique if available. Since no difference could be observed between these approaches (data not shown), only the results based on all acceptable trials from each infant are presented.

\section{Results}

Measurements were performed in 34 infants. In 8 of these infants, satisfactory results could only be obtained for either the SOT $(n=5)$ or the DOT $(n=3)$, whereas in a further 8 infants, no acceptable data were obtained, primarily due to arousal. Technically satisfactory results from both tests were thus achieved in 18 infants (9 female, 9 male) aged 4-41 (median 7.4) weeks and weighing $2.7-9.9 \mathrm{~kg}$ (median $5.1 \mathrm{~kg}$ ). Infants in whom the SOT or DOT technique failed $(n=16)$ did not show any significant differences regarding their gestational age, weight at birth, sex or age, weight or length at test, from those in whom satisfactory results were obtained

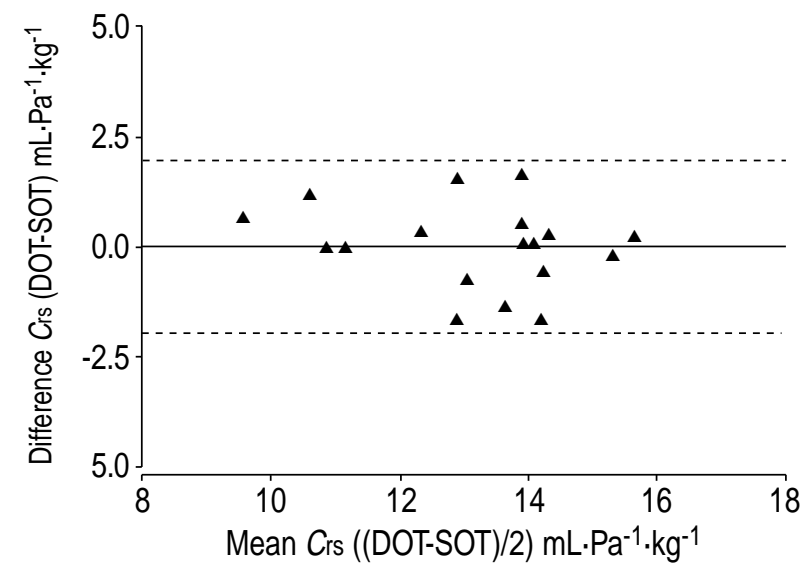

Fig. 3. - Comparison of paired measurements of compliance $(C \mathrm{rs})$ using the Bland and Altman approach [16]: The difference (DOT-SOT) is plotted against the mean compliance ((DOT+ SOT)/2). The continuous line shows the mean difference with $95 \%$ limits of agreement (i.e. $\pm 2 \mathrm{SD})$ shown as dashed lines.

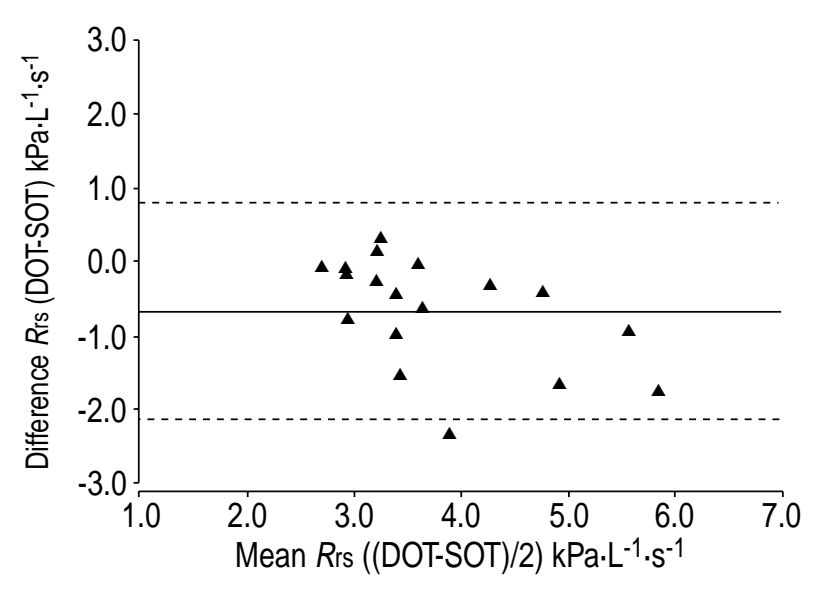

Fig. 4. - Comparison of paired measurements of resistance $\left(R_{\mathrm{rs}}\right)$ : using the Bland and Altman approach [16]: The difference (DOT-SOT) is plotted against the mean resistance ((DOT+ SOT)/2). Continuous line shows the mean difference with $95 \%$ limits of agreement (i.e. $\pm 2 \mathrm{SD})$ as dashed lines.

(data not shown). In those in whom comparisons could be performed, the number of available manoeuvres ranged from 2-14 (median 5) for the SOT and 2-10 (median 4) for the DOT, with $\geqslant 3$ manoeuvres being available for each technique in all but 3 infants.

There were no significant within-subject differences in $C_{\text {rs }}$ according to the technique used, the mean (SD) being 13.17 (1.84) and 13.11 (1.61) $\mathrm{mL} \cdot \mathrm{kPa}^{-1} \cdot \mathrm{kg}^{-1}$ for $C \mathrm{rs}$,SO and $C$ rs,DO, respectively. The $95 \%$ confidence interval (CI) of DOT-SOT was $-0.55-+0.42 \mathrm{~mL} \cdot \mathrm{kPa}^{-1} \cdot \mathrm{kg}^{-1}$ suggesting that the two approaches could be used interchangeably (fig. 3). However, mean (SD) Rrs,DO (3.44 (0.80) $\mathrm{kPa}^{\left.-\mathrm{L}^{-1} \cdot \mathrm{s}\right)}$ was significantly lower than mean (SD) Rrs,SO (4.12 (1.17) $\left.\mathrm{kPa} \cdot \mathrm{L}^{-1} \cdot \mathrm{s}\right)$; the $95 \% \mathrm{CI}$ of this difference being $-1.04--0.31 \mathrm{kPa} \cdot \mathrm{L}^{-1} \cdot \mathrm{s}, \mathrm{p} \leqslant 0.001$ (fig. 4). This difference was greatest in the younger infants with highest mean Rrs (i.e. $\geqslant 3.5 \mathrm{kPa} \cdot \mathrm{L}^{-1} \cdot \mathrm{s}$ ). There was no significant difference in airway pressure during the first plateau, 
$P 1$, the mean (SD) being $0.85 \mathrm{kPa}(0.14)$ for SOT and $0.85 \mathrm{kPa}(0.15)$ for DOT, $(95 \% \mathrm{CI}-0.03-+0.05)$.

\section{Discussion}

The results from this study suggest that, providing strict criteria with respect to technical acceptability are applied, similar values for $C$ rs can be obtained using two different approaches, SOT and DOT, whereas values of $R$ rs,DO are significantly lower than those of $R \mathrm{rs}, \mathrm{SO}$.

\section{Study design and technical aspects}

A major restriction of this study was the fact that since the methodological evaluation of new techniques can never justify the sedation of infants by itself, all comparisons had to be attempted in infants who had been recruited to an epidemiological study. The comparative measurements therefore had to be performed at the end of a full lung function protocol, with infants more likely to wake up than if these tests had been applied as part of a routine protocol. The high failure rate of $47 \%$ for paired measurements reported in this study does not therefore represent a true estimate of the failure rate of the two techniques themselves. Exclusion due to alinearity of the flow/volume curve was only necessary in three of 34 healthy infants in this study. In these cases, a pattern of persistent expiratory braking lead to a convex-flow/volume loop during expiration. Alinearity may be a greater problem in younger infants or in those with respiratory disease in whom a concave flow/volume curve would be expected in presence of expiratory airflow limitation.

During clinical or research studies, the present authors would always attempt to achieve $\geqslant 5$ technically satisfactory manoeuvres for the SOT and only to report a result if a minimum of 3 such trials had been obtained [17, 18]. However, bearing in mind the time constraints described earlier, and the fact that results from individual infants were not being reported for clinical or epidemiological research purposes, data were included for comparison in this study if $\geqslant 2$ acceptable trials within $10 \%$ were available for each test. This compromise was only necessary in 3 of the infants reported and did not appear to bias the results presented in any way.

The biggest impact on increasing the success rate of measurements in infants who remained asleep was the achievement of better automated default settings for factors such as optimum timing and duration of the occlusions. There is limited guidance in the existing literature regarding optimal duration of airway occlusions during measurements of passive mechanics [19]. While an experienced user with manual control of the shutter can usually judge very effectively when both equilibration and relaxation have occurred, it is far more difficult to develop suitable algorithms that will be robust enough to cope with the wide variety of breathing patterns encountered during infant lung function testing. To ensure complete equilibration of pressures within the respiratory system, a minimum occlusion time is required, which in our experience generally proved to be $\geqslant 400 \mathrm{~ms}$. When occlusions shorter than this are allowed, false plateaux which underestimate true recoil pressure may be detected, particularly in infants with airway obstruction or marked expiratory braking, with any subsequent data from the occlusion being lost due to premature shutter opening. By contrast, it is also essential to set a maximum occlusion time to avoid the potential reactivation of the respiratory muscles [20]. During this study it was recognised that the majority of infants need and tolerate a minimum occlusion time of $400 \mathrm{~ms}$ up to a maximum of $1200 \mathrm{~ms}$ to achieve an acceptable first pressure plateau and an occlusion of between 250 and $800 \mathrm{~ms}$ to achieve a satisfactory second plateau. In most infants, the pressure plateau has been seen to develop much faster during the second occlusion, when the respiratory system was already relaxed [6]. This, together with the need to release the occlusion before premature inspiratory efforts occurred, resulted in a minimum plateau duration of $70 \mathrm{~ms}$ rather than $100 \mathrm{~ms}$ being accepted for the second plateau, which again proved suitable in the majority of infants studied. The precise timing of the second occlusion also proved to be absolutely critical to the success of this technique. Sufficient time must elapse after release of the first occlusion to permit appreciable changes in volume and pressure. Yet, if the second occlusion occurs at a lung volume less than $3-4 \mathrm{~mL} \cdot \mathrm{kg}^{-1}$ above the infant's EEL, there is little chance of achieving respiratory muscle relaxation: the commonest responses being active expiration, (with overestimation of $P_{2}$ and underestimation of $C \mathrm{rs}, \mathrm{DO})$ or immediate inspiratory effort with no plateau at all. This is presumably due to the marked volume dependency of the Hering-Breuer inflation reflex over the tidal range [21]. Measurements for the DOT therefore proved to be most successful if the first occlusion was performed at end-inspiration and the second after approximately $50 \%$ of the tidal volume had been expired.

One great advantage of automated equipment to assess infant respiratory function is the reduction of interobserver variability. However, since it is very difficult to develop algorithms that have the same discriminatory powers as the trained human eye, especially where dealing with a wide range of breathing patterns, a close inspection of the collected data is essential. All automated programs should therefore provide the opportunity both to inspect the data prior to acceptance and to perform off-line analysis if necessary.

Causes of the observed discrepancies in respiratory system resistance

Potential causes of the lower values observed for $R$ rs when assessed using the DOT could include: 1) Overestimation of $R \mathrm{rs}, \mathrm{SO}$ due to an overestimation of the $t$ rs. This is, however, highly unlikely, since it would have been accompanied by an overestimation of $C_{\mathrm{rs}, S O}$ with respect to the DOT, which was not observed. 2) Overestimation of $R$ rs,SO due to overestimation of $P 1$. Again, this is an unlikely scenario, 
since it would have been accompanied by an underestimation of $C$ rs,SO with respect to $C$ rs,DO. In addition there were no significant within-subject differences in the values obtained for $P 1$ from the SOT and DOT. 3 ) An underestimation of $P 2$ during the DOT, possibly due to failure to reach equilibrium during the shorter airway occlusion. This would also have been reflected in the difference in $C \mathrm{rs}$, the $C \mathrm{rs}$ obtained from the DOT being lower than that from the SOT due to overestimation of $\Delta P$. 4) An overestimation of flow immediately prior to the second occlusion. The DOT is based on the assumption that the recoil pressure during the second occlusion represents the driving pressure controlling the flow immediately preceding the occlusion. However, in the presence of a slowly closing shutter, there could be significant loss of flow and volume from the system resulting in a relative overestimation of $V^{\prime} 2$. This possibility was excluded by examining ASCII data and recalculating the results manually. This demonstrated that extrapolation of the flow to the point where the plateau had developed would only account for mean decrease in flow of $3 \%$ in the current study. 5) A true physiological difference between $R$ rs measured by the 2 techniques. If the discrepancies cannot be attributed to technical reasons, they may simply reflect the intrinsic variability of resistance measurements, since it is well known that no single value of resistance will be representative for any given infant $[18,22,23]$. The significantly lower $R r s$ measured when using DOT may reflect a degree of volume dependency of $R$ rs values, since $R$ rs,SO was measured at a lower average lung volume than $R$ rs,DO in this study. During the SOT, Rrs was derived from the $t_{\mathrm{rs}} / \mathrm{Crs}$, with $t_{\mathrm{rs}}$ being calculated over a portion of the descending flow/volume loop ranging 55\%-5\% (average 30\%) of the volume remaining in the lung above EEL. By contrast, during the DOT, the second occlusion was performed when $50 \%$ of the tidal volume remained in the lung. The relative narrowing of airway calibre at lower lung volumes, due to decreased transpulmonary pressure, may be sufficient to explain the relatively higher values of $R$ rs when performing SOT. This may also explain the tendency for increased difference between $R$ rs SOT and $R$ rs DOT in the younger infants with higher resistance (fig. 4).

\section{Clinical relevance of findings}

This study only addressed methodological aspects of this new adaptation of the occlusion technique and demonstrated that, by careful adjustment of the timing and duration of the occlusions, it is feasible to use the DOT to measure passive mechanics in infants. However, before the clinical relevance of this new approach can be assessed, far more measurements in both healthy children and those with respiratory disease will need to be performed. In the presence of airway disease, both respiratory frequency and passive equilibration times may be increased. Both factors will make it more difficult to achieve satisfactory plateaux in either technique. Similarly, the assumption that the respiratory system can be described by a single time constant, which is fundamental to accurate measurements using the SOT, is less likely to be valid in the presence of lung disease. Consequently, differences between the two techniques may be more pronounced in infants with disease than found in the current study. In addition, more work is required to establish the intra-subject variability of both techniques in healthy and sick infants, and the intersubject variability, once factors such as body size, age and sex have been taken into account [24].

Only once such work is complete will it be possible to determine the clinical relevance of the DOT, or indeed whether the addition of DOT is more helpful than simply repeating a series of SOT measurements in order to increase confidence in reported results.

\section{Conclusions}

The close agreement between both methods of assessing $C$ rs in healthy infants suggests that the criteria adhered to in this study with respect to technically acceptable data were valid and would provide a useful baseline when extending such measurements to those with lung disease. The comparison of the $R$ rs values obtained by SOT and DOT, respectively, is more complex as there is a significant difference of $R$ rs when using the two methods, even in healthy infants.

Further work is needed to investigate the potential clinical or epidemiological usefulness of this new approach and whether there are any advantages of performing the double occlusion technique as well as, or instead of, the single occlusion technique.

\section{References}

1. Le Souëf PN, England SJ, Bryan AC. Passive respiratory mechanics in newborns and children. $A m$ Rev Respir Dis 1984; 129: 552-556.

2. Mortola JP, Saetta M. Measurements of respiratory mechanics in the newborn: a simple approach. Pediatr Pulmonol 1987; 3: $123-130$.

3. Fletcher ME, Baraldi E, Steinbrugger B. Passive Respiratory Mechanics. In: Stocks J, Sly PD, Tepper RS, Morgan WJ, eds. Infant Respiratory Function Testing. New York, John Wiley \& Sons, Inc., 1996; $283-328$.

4. Olinsky A, Bryan AC, Bryan MH. A simple method of measuring total respiratory system compliance in newborn infants. $S$ Afr Med J 1976; 50: $128-130$.

5. Gappa M, Rabbette PS, Costeloe KL, Stocks J. Assessment of passive respiratory compliance in healthy preterm infants: A critical evaluation. Pediatr Pulmonol 1993; 15: 304-311.

6. Fletcher ME, Dezateux CA, Stocks J. Respiratory compliance in infants - a preliminary evaluation of the multiple interrupter technique. Pediatr Pulmonol 1992; 14: $118-125$.

7. Mortola JP, Hemmings G, Matsuoka T, Saiki C, Fox G. Referencing lung volume for measurements of respiratory system compliance in infants. Pediatr Pulmonol 1993; 16: $248-253$.

8. Morris MG. A simple new technique to measure the effective dead space of the face mask with a water 
volumeter in infants. Eur Respir J 1999; 14: $1163-$ 1166.

9. Frey U, Stocks J, Coates A, Sly P, Bates J. Specifications for equipment used for infant pulmonary function testing. Eur Respir J 2000; 16: 731 - 740 .

10. Squeeze - software for the analysis of lung function test recordings, v1.44. (computer program). Dixon P. \& Stocks J. Imperial College of Science, Technology and Medicine: 1997; 1-46.

11. Henschen M, Stocks J, Hoo AF, Dixon P. Analysis of forced expiratory manoeuvers from raised lung volumes in preterm infants. $J$ Appl Physiol 1998; 85: $1989-1997$.

12. Lum S, Hoo AF, Dezateux C, et al. Identification of small for gestational age infants - which centiles should we be using? Early Hum Dev 1999; 55: 189 - 190.

13. Prechtl HFR. The behavioural states of the newborn infant (a review). Brain Res 1974; 76: 185-212.

14. Stocks J, Nothen U, Sutherland P, Hatch DJ, Helms P. Improved accuracy of the occlusion technique for assessing total respiratory compliance in infants. Pediatr Pulmonol 1987; 3: $71-77$.

15. Bland JM, Altman DG. Comparing methods of measurement: why plotting difference against standard method is misleading. Lancet 1995; 346: $1085-$ 1087.

16. Bland JM, Altman DJ. Statistical methods for assessing agreement between two methods of clinical measurement. Lancet 1986; 307-310.
17. Stocks J, Henschen M, Hoo AF, Costeloe K, Dezateux CA. The influence of ethnicity and gender on airway function in preterm infants. Am J Respir Crit Care Med 1997; 156: $1855-1862$.

18. Dundas I, Dezateux CA, Fletcher ME, Jackson EA, Stocks J. Comparison of single-breath and plethysmographic measurements of resistance in infancy. $\mathrm{Am}$ J Respir Crit Care Med 1995; 151: 1451-1458.

19. Steinbrugger B, Fabian J, Zach MS. The influence of occlusion time on measuring respiratory resistance and compliance in infants with bronchiolitis. Pediatr Res 1993; 33: 273 - 277.

20. England SJ. Current techniques for assessing pulmonary function in the newborn and infant: advantages and limitations. Pediatr Pulmonol 1988; 4: 48-53.

21. Rabbette PS, Stocks J. Influence of volume dependency and timing of airway occlusions on the HeringBreuer reflex in infants. J Appl Physiol 1998; 85: $2033-2039$.

22. Stocks J, Thomson A, Wong C, Silverman M. Pressure-flow curves in infancy. Pediatr Pulmonol 1985; $1: 33-40$.

23. Stocks J, Thomson A, Silverman M. The numerical analysis of pressure-flow curves in infancy. Pediatr Pulmonol 1985; 1: 19-26.

24. Goetz I, Hoo AF, Lum S, et al. Evaluation of the double occlusion technique to measure respiratory resistance in infants. Am J Respir Crit Care Med 2000; 161: A221. 